\title{
The Performance of Step-Wise Group Screening Designs
}

\author{
M.M. Manene and Simwa
}

Department of Mathematics, University of Nairobi, P.O. Box 30197, Nairobi, Kenya, Email: maths@uonbi.ac.ke.

\author{
أذاءفهص عوللل المجموعلت المتتلبعة الظلولت
}

مم. ملن و ر.و.سيموا

\begin{abstract}
خلاصة : هذا البمث بناثش أداء فحص فاعلية مجموعات العولمل المتنابعة الظطوات حيث تحتوي الخطوة الأوله

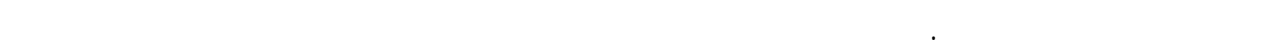

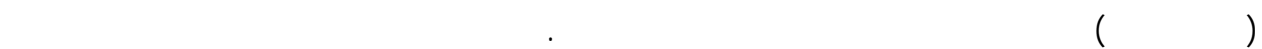

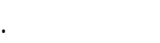

\begin{abstract}
In this paper we evaluate the performance of step-wise group screening designs in which group-factors contain an equal number of factors in the initial step. A usual assumption in group screening designs is that the directions of possible effects are known a-priori. In practice, however, this assumption is unreasonable. We shall examine step-wise group screening designs without errors in observations when this assumption is relaxed. We shall consider cancellations of effects within group-factors. The performance of step-wise group-screening designs shall then be compared with the performance of multistage group screening designs.
\end{abstract}

KEYWORDS: Step-wise designs, group-factors, initial step, subsequent steps, cancellation of effects, screening efficiency.

\section{Introduction}

Tn scientific experimentations situations arise in which a large number of potentially important 1 factors must be examined. In such situations there is often a need, due to limited resources, for an efficient method of factor screening. One such method, introduced by Watson (1961), is the two-stage group screening. This method has been generalized to more than two-stages by Li (1962) and Patel (1962).

The notion of step-wise group screening was introduced by Patel and Manene (1987) basing their method on the group testing procedure introduced by Sterret (1957). Odhiambo and Manene (1987) considered step-wise group screening designs with errors in observations. Manene (1997) extended step-wise group screening designs to what is called "two-type step-wise designs". Manene 
et al. (2002) further extended the two type step-wise group screening designs to multi-type step-wise group screening designs.

A basic assumption in group screening is that the direction of all suspected effects are known or can be corrected a-priori. Under this assumption, factor levels can be assigned in such a way that there is no cancellation of effects within a group-factor. In practice, however, this assumption is inadequate.

Mauro and Smith (1982) considered the performance of two-stage group screening when the assumption of known effect direction is false. Odhiambo (1986) generalized this approach and assessed the performance of multistage group screening when there is a non-zero probability of cancellation of effects within a group-factor.

We shall use the approach, first suggested by Mauro and Smith (1982) and later used by Odhiambo (1986), to assess the performance of step-wise group screening designs. The performance of step-wise group screening designs with cancellation of effects shall then be compared with the performance of multi-stage group screening designs.

\section{Assumptions}

Suppose that $f$ factors are to be screened for their effect on the response. For detecting the factors having major effect, it is usually adequate to assume a first-order linear model.

$$
y_{u}=\beta_{o}+\sum_{i=1}^{f} \beta_{i} x_{u i}+\varepsilon_{u}
$$

where $y_{u}$ is the $\mathrm{u}^{\text {th }}$ response, $\beta_{0}$ is a constant common to every response, $\beta_{i}(i \geq 1)$ is the linear effect of the $i^{\text {th }}$ factor in the $u^{\text {th }}$ run, and $\varepsilon_{u}$ is the $u^{\text {th }}$ error term.

In addition to equation (2.1) assumption, we shall assume that :

(i) All factors have independently the same probability $p_{1}$ of having a positive effect and the same probability $p_{2}$ of having a negative effect. Thus, the probability of a factor being active (defective) is $p=p_{1}+p_{2}$

(ii) All active factors have the same absolute effect, $\Delta \geq 0$, that is

$$
\left|\beta_{i}\right|= \begin{cases}\Delta, & \text { if factor } i \text { is active } \\ 0, & \text { if factor } i \text { is inactive }\end{cases}
$$

(iii) The screening procedure is performed without experimental errors. This means that we take $\varepsilon_{\mathrm{u}}$ $=0$ in equation (2.1).

These are basically the same assumptions made by Mauro and Smith (1982) and Odhiambo (1986).

Suppose that it is desired to classify $f$ factors as active or inactive using the step-wise group screening procedure. The initial step of this procedure consists of dividing the $f$ factors into $g$ firstorder group factors each of size $k(f=k g)$. The first order group factors are then tested for their effect and those found to be effective are set aside. In step two we start with any effective first-order groupfactor and test factors within it one by one till we find an active factor. We set aside factors that are found to be inactive, keeping the active factor separate. In step three, the remaining factors are regrouped in a group, which is then tested for its effect. The test procedure carried out in step one and in step two is repeated successively in the subsequent steps till the analysis terminates with a test on a 
non-effective group-factor or with a group-factor of size one. This test procedure is performed on all group-factors found to be effective in the initial step.

Since there are no experimental errors, it is possible to use designs with the smallest number of runs in the initial step (i.e. the number of runs required to test $g$ group-factors is $g+1$ ), where the one extra run is the control run. This control run may be used at every step of the step-wise design.

\section{The Expected Number of Runs}

Suppose that there are $f$ factors divided into $g$ group-factors in the initial step such that each group-factor contains exactly $k$-factors. Let $X$ denotes the number of factors with positive effects and $Y$ denotes the number of factors with negative effects contained in a group-factor of size $k$ in the initial step. Let

$p_{1}=$ Probability that a factor chosen at random has a positive effect.

$p_{2}=$ Probability that a factor chosen at random has a negative effect.

Then

$$
P(X=x, Y=y)=\left[\begin{array}{l}
\frac{k !}{x ! y !(k-x-y) !} p_{1}^{x} p_{2}^{y}\left(1-p_{1}-p_{2}\right)^{k-x-y,} x+y \leq k \\
0
\end{array}\right.
$$

Otherwise

which is the trinomial distribution with parameters $k_{1}, p_{1}$, and $p_{2}$. This distribution will subsequently be denoted by $\mathrm{B}\left(x ; y ; p_{1}, p_{2}, k\right)$.

At any step of the step-wise design, a group-factor is active if it contains at least one active factor. However, an active group-factor will have a significant effect (i.e. will be effective) if and only if the factor effects do not cancel completely within the group-factor. Let us define

and

$$
p^{*}=P(\text { group at the initial step is active) }
$$

$$
\theta=P \text { ( a group factor at the initial step is effective) }
$$

Then

$$
p^{*}=1-p(x=0, y=0)=1-\left(1-p_{1}-p_{2}\right)^{k}
$$

and

$$
\begin{aligned}
& \quad \theta=1-p(x=y) \\
= & 1-\left(1-p_{1}-p_{2}\right)^{k}-\sum_{x=1}^{k / 2} B\left(x ; y ; p_{1}, p_{2}, k\right) \\
= & p^{*}-\theta^{*}
\end{aligned}
$$

where

$$
\theta^{*}=p^{*}-\theta
$$


is the probability of cancellation within an active group-factor at the initial step. Let $M$ denote the number of active group-factors and $N$ denote the number of effective group-factors in the initial step.

Then,

$$
P(M=m)=\left\{\begin{array}{l}
\left(\begin{array}{l}
g \\
m
\end{array}\right) p^{* m}\left(1-p^{*}\right)^{g-m,} m=0,1, \ldots, g \\
0 \quad \text { otherwise }
\end{array}\right.
$$

and

$$
P(N=n)=\left\{\begin{array}{l}
\left(\begin{array}{l}
g \\
n
\end{array}\right) \theta^{n}(1-\theta)^{g-n} n=0,1, \ldots, g \\
0 \quad \text { otherwise }
\end{array}\right.
$$

Thus

$$
E(M)=g p^{*} \text { and } E(N)=g \theta
$$

Let $p_{k}^{*}(x, y)$ be the probability that a group-factor that has been identified as effective in the initial step contains exactly $x$ factors with positive effects and $y$ factors with negative effects for $x, y=0, \ldots, k, x \neq y$. Then

$$
\begin{aligned}
& p_{k}^{*}(x, y)=\frac{\mathrm{B}\left(x ; y ; p_{1}, p_{2}\right)}{\theta} \\
& =\frac{1}{\theta} \frac{k !}{x ! y !} p_{1}^{x} p_{2}^{y}\left(1-p_{1}-p_{2}\right)^{k-x-y} \quad(x+y \neq 0, x \neq y) .
\end{aligned}
$$

Denote by $\mathrm{E}_{k}\left(R_{(x, y)}\right)(x, y=0,1,2, \ldots, k, x \neq y, 0 \leq x+y<k)$ the expected number of runs required to classify as effective or non-effective factors within a group-factor that was found to be effective at the initial step if it contains $x$ factors with positive effects and $y$ factors with negative effects. If $x \neq 0$ and $y=0$, then $\mathrm{E}_{k}\left(R_{(x, y)}\right)$ reduces to $\mathrm{E}_{k}\left(R_{(x, o)}\right)$ which is actually the same as $\mathrm{E}_{k}\left(R_{x}\right)$ given by Patel and Manene (1987). Thus

$$
E_{k}\left(R_{(x, 0)}\right)=\frac{x k}{x+1}+x+\frac{x}{x+1}-\frac{2 x}{k}, \quad x=1,2, \ldots, k
$$

Similarly if $x=0$ and $y \neq 0$,

$$
\mathrm{E}_{k}\left(R_{(0, y)}\right)=\frac{y k}{y+1}+y+\frac{y}{y+1}-\frac{2 y}{k}, y=1,2, \ldots, k
$$

Thus,

$$
\mathrm{E}_{k}\left(R_{(0, x)}\right)=\mathrm{E}_{k}\left(R_{(x, 0)}\right) \quad, \quad x=y
$$


and that

$$
\mathrm{E}_{k}\left(R_{(x, y)}\right)=\mathrm{E}_{k}\left(R_{(y, x)}\right), \quad x \neq y
$$

However, if $x=y$ (i.e. the number of active factors with positive effects equals the number of active factors with negative effects), then the effects cancel completely and such a group-factor is dropped from further analysis in the initial step. Thus

$$
E_{k}\left(R_{(x, X)}\right)=0, \quad x=0,1,2, \ldots, \frac{k}{2}
$$

Lemma 3.1

$$
\mathrm{E}_{k}\left(R_{(1,2)}\right)=\frac{13}{18} k+\frac{29}{9}-\frac{7}{k}-\frac{7}{3 k(k-1)}
$$

\section{Proof}

There are two cases to consider, namely

(i) the first active factor detected has a positive effect;

(ii) the first active factor detected has a negative effect.

Considering case (i), the probability that the first factor tested has a positive effect is $1 / k$ and the probability that the $(l+1)$-st factor tested is the first active factor and has a positive effect is

$$
\Pi_{w=1}^{l} \frac{k-(w+1)}{k-(w-1)} \frac{1}{k-1}
$$

On average, we shall require $1+1+E_{k-1}\left(R_{(0,2)}\right)$ tests to complete the test procedure if the first factor tested is active and has a positive effect, and $l+1+1+E_{k-(l+1)}\left(R_{(0,2)}\right)$ tests to complete the test procedure if the $(l+1)$-st factor tested is the first active factor and has a positive effect. Considering case (ii), the probability that the first factor tested has a negative effect is $2 / k$ and the probability that the $(l+1)-$ st factor tested is the first active factor and has a negative effect is

$$
\Pi_{w=1}^{l} \frac{k-(w+1)}{k-(w-1)} \frac{2}{k-l}
$$

On the average we shall require $1+1+E_{k-1}\left(R_{(1,1)}\right)$ tests to complete the test procedure if the first factor tested is active and has a negative effect, and $l+1+1+\mathrm{E}_{k-(l+1)}\left(R_{(1,1)}\right)$ tests to complete the test procedure if the $(l+1)$-st factor tested is the first active factor and has a negative effect. It follows that 


$$
\begin{gathered}
\mathrm{E}_{k}\left(R_{(1,2)}\right)=\frac{1}{k}\left\{1+1+\mathrm{E}_{k-1}\left(R_{(0,2)}\right)\right\}+\sum_{l=1}^{k-3} \Pi_{w=1}^{l} \frac{k-(w+1)}{k-(w-1)} \frac{1}{w-1} \frac{1}{k-l} \frac{1}{2} \\
x\left(l+1+1+\mathrm{E}_{k-(l+1)}\right)\left(R_{(0,2)}\right)+\frac{2}{k}\left\{1+1+\mathrm{E}_{k-1}\left(R_{(1,1)}\right)\right\} \\
+\sum_{l=1}^{k-3} \Pi_{w=1}^{l} \frac{k-(w+1)}{k-(w-1)} \frac{2}{k-l}\left\{l+1+1+\mathrm{E}_{k-1}\left(R_{(1,1)}\right)\right\}
\end{gathered}
$$

Rewriting equation (3.12) and using results in equation (3.9) and equation (3.10) and expanding the resulting expression we obtain (3.11).

This completes the proof of the lemma.

\section{Lemma 3.2}

$$
\mathrm{E}_{k}\left(R_{(1,3)}\right)=\frac{47}{60} k+\frac{529}{120}-\frac{9}{k}-\frac{7}{2 k(k-1)}
$$

Proof. We again consider the two cases highlighted in the proof of lemma 3.1 above. Considering case (i), the probability that the first factor tested has a positive effect is $1 / k$ and the probability that the $(l+1)$ - st factor tested is the first active factor and has a positive effect is

$$
\Pi_{w=1}^{l} \frac{k-(w+3)}{k-(w-1)} \frac{1}{k-l} .
$$

On average we shall need $1+1+\mathrm{E}_{k-1}\left(R_{(0,3)}\right)$ tests to complete the test procedure if the first factor tested is active and has a positive effect, and $l+1+1+E_{k-(l+1)}\left(R_{(0,3)}\right)$ tests to complete the test procedure if the $(l+1)$-st factor tested is the first active factor and has a positive effect.

For case (ii), the probability that the first factor tested has a negative effect is $3 / k$ and the probability that the $(l+1)$ - st factor tested is the first active factor and has a negative effect is

$$
\Pi_{w=1}^{l} \frac{k-(w+3)}{k-(w-1)} \frac{3}{k-l} .
$$

On the average we shall need $1+1+\mathrm{E}_{k-1}\left(R_{(1,2)}\right)$ tests to complete the test procedure if the first factor tested is active and has a negative effect; and $l+1+1+E_{k-(l+1)}\left(R_{(1,2)}\right)$ tests to complete the rest procedure if the $(l+1)$-st factor tested is the first active factor and has a negative effect. Thus 


$$
\begin{aligned}
& \mathrm{E}_{k}\left(R_{(1,3)}\right)=\frac{1}{k}\left(1+1+\mathrm{E}_{k-1}\left(R_{(0,3)}\right)\right)+\sum_{l=1}^{k-4} \Pi_{w=1}^{l} \frac{k-(w+3)}{k-(w-1)} \frac{1}{k-l} \\
& x\left(l+1+1+\mathrm{E}_{k-(l+1)}\left(R_{(0,3)}\right)\right)+\frac{3}{k}\left(1+1+\mathrm{E}_{k-1}\left(R_{(1,2)}\right)\right) \\
& \quad+\sum_{l=1}^{k-4} \Pi_{w=1}^{l} \frac{k-(w+3)}{k-(w-1)} \frac{3}{k-l}\left(l+1+1+\mathrm{E}_{k-(l+1)}\left(R_{(1,2)}\right)\right)
\end{aligned}
$$

Using results in equation (3.9) and lemma 3.1 in equation (3.14), expanding the resulting expression and simplifying we obtain (3.13).

This completes the proof of lemma 3.2.

Using the same argument as that used in the proof of lemma 3.1 and lemma 3.2, we obtain

$$
\begin{aligned}
& \mathrm{E}_{k}\left(R_{(1,4)}\right)=\frac{37}{45} k+\frac{497}{90}-\frac{11}{k}-\frac{14}{3 k(k-1)} \\
& \mathrm{E}_{k}\left(R_{(2,3)}\right)=\frac{77}{180} k+\frac{259}{90}-\frac{9}{2 k}-\frac{7}{3 k(k-1)} \\
& E_{k}\left(R_{(1,5)}\right)=\frac{107}{126} k+\frac{1663}{252}-\frac{13}{k}-\frac{35}{6 k(k-1)} \\
& \text { and } \quad \mathrm{E}_{k}\left(R_{(2,4)}\right)=\frac{28}{45} k+\frac{217}{45}-\frac{8}{k}-\frac{14}{3 k(k-1)}
\end{aligned}
$$

Let $R_{s}^{0}$ denotes the number of tests required to analyse a group-factor of size $k$ that is known to be effective.

$$
\mathrm{E}\left(R_{s}^{o}\right)=\sum_{x=0}^{k} \sum_{y=0}^{k} E_{k}\left(R_{(x, y)}\right) p_{k}^{*}(x, y)
$$

Then

$$
\left.=\sum_{x=0}^{k} \sum_{y=0}^{k} \mathrm{E}_{k}\left(R_{(x, y)}\right) \frac{B\left(x ; y ; p_{1}, p_{2}, k\right)}{\theta}(x+y \leq k)_{(x+y \leq k}\right)
$$

Denote by $R_{s}$ the number of tests required to analyse all the factors in the $\mathrm{N}$ group-factors found to be effective in the initial step. Then

$$
R_{s}=N E\left(R_{s}^{0}\right)
$$

Let $R$ be the total number of runs required to analyse the $f$ factors under investigation, then

$$
R=R_{1}+R_{2}
$$

where $\quad R_{I}=1+\frac{f}{k}$ is the number of runs in the initial step.

Using (3.6), (3.19) and (3.20) in (3.21) and taking the expected value on both sides we obtain. 


$$
E(R)=1+\frac{f}{k}+\frac{f}{k} \sum_{x=0}^{k} \sum_{y=0}^{k} E_{k}\left(R_{(x, y)}\right) B\left(x ; y ; p_{1}, p_{2}, k\right)(\mathrm{x}+\mathrm{y} \leq \mathrm{k})
$$

Theorem 3.1 Let $R$ denote the total number of runs required to classify as effective or non-effective all the $f$ ' factors under investigation in a step-wise group screening experiment. Then

$$
\begin{gathered}
\mathrm{E}(R) \approx 1+\frac{f}{k}+\frac{f}{k}\left[\sum_{j=1}^{4} \frac{k(k-1) \ldots(k-j+1)}{j !}(1-p)^{k-j}\left(p_{1}^{j}+p_{2}^{j}\right)\right. \\
\left.x\left(\frac{j}{j+1} k+j+\frac{j}{j+1}-\frac{2}{k} j\right)\right]+\frac{k-2}{15120} \frac{f}{k} p_{1} p_{2}\left(420\left(13 k^{3}+45 k^{2}-184 k+84\right) p(1-p)^{k-3}\right. \\
\left.+21(k-3)\left(94 k^{3}+435 k^{2}-1609 k+660\right)\left(p_{1}^{2}+p_{2}^{2}\right)(1-p)^{k-4}\right)
\end{gathered}
$$

where $p_{1}$ is the a-priori probability of a factor being effective with positive effect and $p_{2}$ is the a-priori probability of a factor being effective with negative effect $\left(p=p_{1}+p_{2}\right)$, and $k$ is the size of the groupfactor at the initial step.

Proof. From (3.22) we have

$$
\mathrm{E}(R)=1+\frac{f}{k}+\frac{f}{k} \sum_{x=0}^{k} \sum_{y=0}^{k} E_{k}\left[R_{(x, y)}\right] B\left(x ; y ; p_{1}, p_{2}, k\right) \quad(x+y \leq k)
$$

Since $p$, the apriori probability of a factor being effective is usually small, the probability of having more than four effective factors in an effective group factor is negligible. We can thus assume that an effective group-factor will not have more than four effective factors. Using this assumption we have

$$
\begin{gathered}
\mathrm{E}(R) \approx 1+\frac{f}{k}+\frac{f}{k}\left[\sum _ { j = 1 } ^ { 4 } ( \frac { j k } { j + 1 } + j + \frac { j } { j + 1 } - \frac { 2 j } { k } ) \left[B\left(0 ; j ; p_{1}, p_{2}, k\right)\right.\right. \\
\left.\left.+\mathrm{B}\left(j ; 0 ; p_{1}, p_{2}, k\right)\right]+\sum_{x=1}^{4} \sum_{y=1}^{4} \mathrm{E}_{k}\left[R_{(x, y)}\right] B\left(x ; y ; p_{1}, p_{2}, k\right)\right] \\
(x+y \leq 4, x \neq y)
\end{gathered}
$$

Now

$$
\begin{gathered}
\sum_{x=1}^{4} \sum_{y=1}^{4} \mathrm{E}_{k}\left[R_{(x, y)}\right] B\left(x ; y ; p_{1}, p_{2}, k\right) \\
=\mathrm{E}_{k}\left[R_{(1,2)}\right] B\left(1 ; 2 ; p_{1}, p_{2}, k\right)+\mathrm{E}_{k}\left[R_{(2,1)}\right] B\left(2 ; 1 ; p_{1}, p_{2}, k\right) \\
+\mathrm{E}_{k}\left[R_{(1,3)}\right] B\left(1 ; 3 ; p_{1}, p_{2^{k}}\right)+\mathrm{E}_{k}\left[R_{(3,1)}\right] \mathrm{B}\left(3 ; 1 ; p_{1}, p_{2}, k\right) \\
=\frac{k-2}{15120} p_{1} p_{2}\left(420\left(13 k^{3}+45 k^{2}-184 k+84\right) p(1-p)^{k-3}\right. \\
\left.+21(k-3)\left(94 k^{3}+435 k^{2}-1609 k+660\right)\left(p_{1}^{2}+p_{2}^{2}\right)(1-p)^{k-4}\right) \\
(x+y \leq 4, \quad x \neq y, x+y \leq k)
\end{gathered}
$$


using (3.12), (3.14) and noting that $\mathrm{E}_{k}\left(R_{(x, y)}\right)=\mathrm{E}_{k}\left(R_{(y, x)}\right)$ and that $p=p_{1}+p_{2}$.

The proof of the theorem follows from using results in equation (3.24) in equation (3.23) and simplifying the resulting expression.

\section{Expected Number of Active Factors Detected}

Let $A_{I}$ denote the number of active factors within active group-factors declared non-effective in the initial step. Then

$$
A_{\mathrm{I}}=(M-N) k p
$$

Denote by $E_{k}\left[I_{(x, y)}\right]$ the expected number of active factors declared inactive from among the $\mathrm{k}$ factors within a group-factor which was classified as effective in the initial step if it contains $x$ factors with positive effects and $y$ factors with negative effects. Obviously

$$
\begin{aligned}
& E_{k}\left[\mathrm{I}_{(x, o)}\right]=\mathrm{E}_{k}\left[\mathrm{I}_{(0, y)}\right]=0 \\
& \mathrm{E}_{k}\left[\mathrm{I}_{(x, y)}\right]=\mathrm{E}_{k}\left[I_{(y, x)}\right], \quad x \neq y
\end{aligned}
$$

To obtain $\mathrm{E}_{k}\left[\mathrm{I}_{(x, y)}\right], x \neq y, x, y=1,2, \ldots, k, x+y \leq k$; We consider the order in which the active factors occur for given $x$ and $y$ and the corresponding number of undetected active factors in each case. As an illustration, consider the case when $x=1$ and $y=2$. Then the possible orders in which active factors appear are as indicated in Table I using '- ' for negative effect and '+' for positive effect.

Table 1. Determining $\mathrm{I}_{(1,2)}$

\begin{tabular}{|lll|l|}
\hline \multicolumn{3}{|c|}{ Possibilities } & $\mathrm{I}_{(1,2)}$ \\
\hline- & - & + & 2 \\
- & + & - & 2 \\
+ & - & - & 0 \\
\hline
\end{tabular}

Thus

Similarly

$$
\mathrm{E}_{k}\left[\mathrm{I}_{(1,2)}\right]=2 \times \frac{2}{3}+0 \times \frac{1}{3}=\frac{4}{3}
$$

$\mathrm{E}_{k}\left[\mathrm{I}_{(1,3)}\right]=1, \mathrm{E}_{k}\left[\mathrm{I}_{(1,4)}\right]=\frac{4}{5}, \mathrm{E}_{k}\left[\mathrm{I}_{(2,3)}\right]=\frac{14}{5}, \mathrm{E}_{k}\left[\mathrm{I}_{(1,5)}\right]=\frac{2}{3}$ and $\mathrm{E}_{k}\left[\mathrm{I}_{(2,4)}\right]=\frac{32}{15}$

Let $\bar{A}_{s}^{0}$ denote the number of undetected active factors in a group-factor of size $k$ that were found to be effective in the initial step. Then

$$
\mathrm{E}\left[\bar{A}_{s}^{o}\right]=\sum_{x=0}^{k} \sum_{y=0}^{k} \mathrm{E}_{k}\left[\mathrm{I}_{(x, y)}\right] p_{k}^{*}(x, y)(x+y \leq k) x \neq y
$$


Denote by $\overline{A_{S}}$ the number of undetected active factors within the $N$ group-factors of size $k$ found to be effective in the initial step. Further let $\bar{A}$ denote the total number of undetected active factors from among the $f$ factors under investigation. Then

$$
\mathrm{A}_{s}=N E\left[\bar{A}_{s}^{o}\right]
$$

and

$$
\bar{A}=\bar{A}_{I}+\bar{A}_{s}
$$

Thus

$$
\mathrm{E}[\bar{A}]=\mathrm{E}\left[\bar{A}_{\mathrm{I}}\right]+\mathrm{E}\left[\bar{A}_{s}\right]
$$

Using results in equations (3.3), (3.6), (4.1), (4.6) and (4.7) in equation (4.9) we obtain

$$
\mathrm{E}[\bar{A}]=f p \theta^{*}+\frac{f}{k} \sum_{x=0}^{k} \sum_{y=0}^{k} \mathrm{E}_{k}\left[\mathrm{I}_{(x, y)}\right] \mathrm{B}\left(x ; y ; p_{1}, p_{2}, k\right) \quad(x+y \leq k)
$$

Theorem 4.1 Let $\bar{A}$ denote the total number of undetected active factors in a step-wise group screening design in which an active factor has either a positive effect or a negative effect with respective probabilities $p_{1}$ and $p_{2}\left(p_{1}+p_{2}=p\right)$. Then

$$
\mathrm{E}(\overline{\mathrm{A}})=f p \theta^{*}+\frac{(k-1)(k-2)}{6} f p_{1} p_{2}\left[4 p(1-p)^{k-3}+(k-3)\left(p_{1}^{2}+p_{2}^{2}\right)(1-p)^{k-4}\right]
$$

where $f$ is the number of factors under investigation, $k$ is the size of each group-factor at the initial step and $\theta^{*}$ is the probability of cancellation within a group-factor of size $k$.

Proof. Equation (4.10) gives

$$
\mathrm{E}[\overline{\mathrm{A}}]=f p \theta^{*}+\frac{f}{k} \sum_{x=o}^{k} \sum_{y=0}^{k} \mathrm{E}_{k}\left[\mathrm{I}_{(x, y)}\right] \mathrm{B}\left(x ; y ; p_{1}, p_{2}, k\right) \quad(x+y \leq k) .
$$

If we assume that at an initial step a group-factor contains no more than four active factors and using equations (4.4) and (4.5) and simplifying we obtain equation (4.11).

This completes the proof of theorem (4.1).

Let $A$ be the total number of active factors that are detected in a step-wise group screening design when cancellation is allowed. Then,

$$
\begin{aligned}
\mathrm{E}(\mathrm{A}) & =f p-\mathrm{E}(\overline{\mathrm{A}}) \\
& \approx f p-\left\{f p \theta^{*}+\frac{(k-1)(k-2)}{6} f p_{1} p_{2}\left[4 p(1-p)^{k-3}+(k-3)\left(p_{1}^{2}+p_{2}^{2}\right)(1-p)^{k-4}\right]\right\}
\end{aligned}
$$

where $f p$ is the expected total number of active factors among the $f$ factors under investigation. When either $p_{1}=0$ or $p_{2}=0, \theta^{*}$ will also be zero and $E(A)=f p$.

\section{Comparison of Relative Performance of Step-Wise Group Screening and S- stage Group Screening $(s=2,3)$}

To measure the efficiency of a step-wise group screening design, we need to obtain the efficiency of detecting active factors and the relative testing cost. Let us define 


$$
\phi_{A}=\frac{100 \mathrm{E}(\bar{A})}{f p}
$$

as a percentage measure of the efficiency of a step-wise group screening strategy for detecting the active factors. As another measure of the efficiency of a step-wise group screening procedure we define the relative testing cost,

$$
\mathrm{E}_{R}=\frac{100 \mathrm{E}(R)}{f+1}
$$

as the ratio, expressed as a percentage, of the expected number of runs required by step-wise group screening design to the number of runs required to test the factors individually.

A large value of $\phi_{A}$ or a smaller value of $E_{R}$ indicates better performance on the average, but both measures should be considered in assessing the performance of a group screening strategy to ensure lack of conflict. Only if one group screening design has both a large $\phi_{A}$ and a smaller $E_{R}$ than another screening strategy can the first be said to be definitely better than the second.

Expressions for $E(R)$ and $E(\overline{\mathrm{A}})$ for the step-wise group screening design allowing for cancellations are given in theorem 3.1 and equation (3.17) respectively. Odhiambo (1986) gave the corresponding expressions for an s-stage group screening design as

$$
\mathrm{E}\left(R_{s}\right)=1+g+\sum_{r=2}^{s-1} \Pi_{j=1}^{r} g_{j} \theta_{r-1}+f \theta_{s-1}
$$

and

$$
\mathrm{E}\left(\overline{\mathrm{A}}_{s}\right)=f p\left(1-\sum_{r=1}^{s-1} \theta_{r}^{*}\right)
$$

respectively, where $g_{j}(j=1,2, \ldots, s-1)$ is the number of the $j^{\text {th }}$ order roup-factors, $\theta_{r}(r=1,2, \ldots, s-1)$ is the probability that an $r^{\text {th }}$ order group-factor is effective and $\theta_{r}^{*}$ is the probability of cancellation in an $\mathrm{r}^{\text {th }}$ order group-factor $(r=1,2, \ldots, \mathrm{s}-1, \mathrm{~s} \geq 2)$.

Table 2 shows the relative performance of step-wise group screening designs as compared with the two-stage group screening designs under different partitions of $p$ 's. For both designs, for a particular value of $p$, if $p_{1}=p_{2}=p / 2$, the minimum expected number of runs are slightly fewer and the efficiency in detecting effective factors is slightly lower than when $p_{1} \neq p_{2}$. The difference in the minimum expected number of runs and in the efficiency in detecting effective factors increases as the difference between $p_{1}$ and $p_{2}$ becomes larger. It should be noted that $p_{2}=p-p_{1}$. For all partitions, step-wise group screening has fewer runs than the corresponding two-stage group-screening design. Step-wise group screening designs outperforms two-stage group screening designs for values of $p \geq 0.08$ for all partitions of $p$ 's. That is for $p \geq 0.8$ step-wise group screening designs have both fewer number of runs and are more efficient in detecting active factors than the two-stage group screening designs.

From Table 3 we again observe that for the three stage group screening designs, the minimum expected number of runs for a particular value of $p$ are slightly fewer when $p_{1}=p_{2}=p / 2$ and increases as the difference between increases $p_{1}$ and $p_{2}$. Also the efficiency in detecting effective 
factors increases as the difference between $p_{1}$ and $p_{2}$ for fixed $p$ increases. For all the partitions, step-wise group screening designs are more efficient in detecting active factors than three-stage group screening designs. Step-wise group screening designs outperforms the three-stage group screening design for $p \geq 0.05$.

In both Tables the horizontal lines indicate the beginning of a different partition. It should be noted that Tables 2 and 3 are only for illustration and are not exhaustive.

Table 2. Relative performance of step-wise and two-stage group screening designs for $f=100$ and specified values of $p_{1}$ and $p_{2}$.

\begin{tabular}{|c|c|c|c|c|c|c|c|c|}
\hline \multirow[b]{2}{*}{$p$} & \multirow[b]{2}{*}{$p_{1}$} & \multirow[b]{2}{*}{$p$} & \multicolumn{3}{|c|}{$\begin{array}{l}\text { Step-wise group } \\
\text { screening }\end{array}$} & \multicolumn{3}{|c|}{ Two stage group screening } \\
\hline & & & $k$ & $\phi_{\mathrm{A}}$ & $\phi_{\mathrm{A}}$ & $k_{1}$ & $\operatorname{Min} . E(R)$ & $\phi$ \\
\hline 0.001 & 0.0005 & 0.0005 & 46 & 5.56 & 99.94 & 32 & 7.25 & 99.98 \\
\hline 0.002 & 0.00 & 0.001 & 33 & 7.49 & 99.89 & 23 & 9.80 & 99.95 \\
\hline 0.004 & 0.00 & 0.002 & 23 & 10.29 & 99.79 & 17 & 13.37 & 99.90 \\
\hline 0.005 & 0.002 & 0.0025 & 21 & 11.43 & 99.73 & 15 & 14.79 & 99.88 \\
\hline 0.008 & 0.00 & 0.004 & 16 & 14.35 & 99.62 & 12 & 18.33 & 99.81 \\
\hline 0.009 & 0.0045 & 0.0045 & 16 & 15.20 & 99.53 & 11 & 19.35 & 99.79 \\
\hline 0.01 & 0.005 & 0.005 & 15 & 16.01 & 99.50 & 11 & 20.31 & 99.75 \\
\hline 0.03 & 0.015 & 0.015 & 8 & 28.12 & 98.92 & 7 & 33.67 & 99.19 \\
\hline 0.04 & 0.02 & 0.02 & 7 & 32.73 & 98.92 & 6 & 38.37 & 98.98 \\
\hline 0.05 & 0.025 & 0.025 & 6 & 36.89 & 98.50 & 5 & 42.55 & 98.93 \\
\hline 0.06 & 0.03 & 0.03 & 6 & 40.76 & 97.92 & 5 & 46.11 & 98.50 \\
\hline 0.07 & 0.035 & 0.035 & 5 & 44.16 & 97.76 & 5 & 49.46 & 98.03 \\
\hline 0.08 & 0.04 & 0.04 & 5 & 47.53 & 97.64 & 5 & 52.59 & 97.50 \\
\hline 0.002 & 0.0012 & 0.0008 & 33 & 7.50 & 99.89 & 23 & 9.80 & 99.95 \\
\hline 0.004 & 0.0024 & 0.0016 & 23 & 10.29 & 99.80 & 17 & 13.37 & 99.90 \\
\hline 0.005 & 0.003 & 0.002 & 21 & 11.43 & 99.74 & 15 & 14.79 & 99.88 \\
\hline 0.008 & 0.0048 & 0.0032 & 16 & 14.35 & 99.64 & 12 & 18.33 & 99.81 \\
\hline 0.009 & 0.0054 & 0.0036 & 16 & 15.21 & 99.55 & 11 & 19.36 & 99.80 \\
\hline 0.01 & 0.006 & 0.004 & 15 & 16.02 & 99.52 & 11 & 20.32 & 99.76 \\
\hline 0.03 & 0.018 & 0.012 & 8 & 28.13 & 98.96 & 7 & 33.71 & 99.22 \\
\hline 0.04 & 0.024 & 0.016 & 7 & 32.73 & 98.67 & 6 & 38.41 & 99.02 \\
\hline 0.05 & 0.03 & 0.02 & 6 & 36.90 & 98.56 & 5 & 42.59 & 98.97 \\
\hline 0.06 & 0.036 & 0.024 & 6 & 40.76 & 98.22 & 5 & 46.17 & 98.56 \\
\hline
\end{tabular}


THE PERFORMANCE OF STEP-WISE GROUP SCREENING DESIGNS

\begin{tabular}{|l|c|c|c|c|c|c|c|c|}
\hline 0.07 & 0.042 & 0.028 & 5 & 44.17 & 98.00 & 5 & 49.54 & 98.10 \\
\hline 0.08 & 0.048 & 0.032 & 5 & 47.5 & 97.73 & 5 & 52.69 & 97.60 \\
\hline 0.001 & 0.000 & 0.0009 & 46 & 5.57 & 99.98 & 32 & 7.27 & 99.99 \\
\hline 0.002 & 0.0002 & 0.0018 & 32 & 7.52 & 99.96 & 23 & 9.83 & 99.98 \\
\hline 0.004 & 0.0004 & 0.0036 & 23 & 10.34 & 99.92 & 16 & 13.43 & 99.97 \\
\hline 0.005 & 0.0005 & 0.0045 & 21 & 11.50 & 99.90 & 15 & 14.87 & 99.96 \\
\hline 0.008 & 0.0008 & 0.0072 & 16 & 14.44 & 99.86 & 12 & 18.45 & 99.93 \\
\hline 0.009 & 0.0009 & 0.0081 & 15 & 15.30 & 99.85 & 11 & 19.48 & 99.93 \\
\hline 0.01 & 0.001 & 0.009 & 15 & 16.11 & 99.81 & 11 & 20.47 & 99.91 \\
\hline 0.03 & 0.003 & 0.027 & 8 & 28.26 & 99.61 & 6 & 34.15 & 99.78 \\
\hline 0.04 & 0.004 & 0.036 & 7 & 32.85 & 99.50 & 6 & 39.02 & 99.63 \\
\hline 0.05 & 0.005 & 0.045 & 7 & 37.02 & 99.35 & 5 & 43.23 & 99.61 \\
\hline 0.06 & 0.006 & 0.054 & 6 & 40.80 & 99.25 & 5 & 47.07 & 99.46 \\
\hline 0.07 & 0.007 & 0.063 & 5 & 44.26 & 99.23 & 5 & 50.72 & 99.29 \\
\hline 0.08 & 0.008 & 0.072 & 5 & 47.62 & 99.15 & 5 & 54.19 & 99.10 \\
\hline
\end{tabular}

Table 3. Relative performance of step-wise and three-stage group screening designs for $f=100$ and specified values of $p_{1}$ and $p_{2}$

\begin{tabular}{|c|c|c|c|c|c|c|c|c|c|}
\hline \multirow[b]{2}{*}{$p$} & \multirow[b]{2}{*}{$p_{1}$} & \multirow[b]{2}{*}{$p_{2}$} & \multicolumn{3}{|c|}{ Step-wise group screening } & \multicolumn{4}{|c|}{ Three stage group screening } \\
\hline & & & $k$ & Min. $E(R)$ & $\phi_{\mathrm{A}}$ & $k_{1}$ & $k_{2}$ & Min. $E(R)$ & $\phi_{\mathrm{A}}$ \\
\hline 0.002 & 0.001 & 0.001 & 33 & 7.49 & 99.98 & 70 & 8 & 5.59 & 99.57 \\
\hline 0.004 & 0.002 & 0.002 & 23 & 10.29 & 99.79 & 44 & 6 & 8.22 & 99.35 \\
\hline 0.005 & 0.0025 & 0.0025 & 21 & 11.43 & 99.73 & 40 & 6 & 9.34 & 99.17 \\
\hline 0.008 & 0.004 & 0.004 & 16 & 14.35 & 99.62 & 30 & 5 & 12.30 & 98.85 \\
\hline 0.009 & 0.0045 & 0.0045 & 16 & 15.20 & 99.53 & 28 & 5 & 13.18 & 98.75 \\
\hline 0.01 & 0.005 & 0.005 & 15 & 16.01 & 99.50 & 27 & 5 & 14.04 & 98.58 \\
\hline 0.03 & 0.015 & 0.015 & 8 & 28.12 & 98.92 & 16 & 4 & 27.22 & 96.18 \\
\hline 0.04 & 0.02 & 0.02 & 7 & 32.73 & 98.62 & 12 & 3 & 32.36 & 95.22 \\
\hline 0.05 & 0.025 & 0.025 & 6 & 36.89 & 98.50 & 12 & 3 & 36.89 & 94.63 \\
\hline 0.06 & 0.03 & 0.03 & 6 & 40.76 & 97.92 & 12 & 3 & 41.05 & 92.95 \\
\hline 0.07 & 0.035 & 0.035 & 5 & 44.16 & 97.76 & 12 & 3 & 44.90 & 91.24 \\
\hline 0.08 & 0.04 & 0.04 & 5 & 47.53 & 97.64 & 12 & 3 & 48.47 & 89.55 \\
\hline
\end{tabular}


M.M. MANENE and R.O. SIMWA

\begin{tabular}{|c|c|c|c|c|c|c|c|c|c|}
\hline 0.002 & 0.0012 & 0.0008 & 33 & 7.50 & 99.89 & 70 & 8 & 5.60 & 99.59 \\
\hline 0.004 & 0.0024 & 0.0016 & 23 & 10.29 & 99.80 & 44 & 6 & 8.22 & 99.37 \\
\hline 0.005 & 0.003 & 0.002 & 21 & 11.43 & 99.74 & 40 & 6 & 9.34 & 99.21 \\
\hline 0.008 & 0.0048 & 0.0032 & 16 & 14.35 & 99.64 & 30 & 5 & 12.31 & 98.90 \\
\hline 0.009 & 0.0054 & 0.0036 & 16 & 15.21 & 99.55 & 28 & 5 & 13.19 & 98.80 \\
\hline 0.01 & 0.006 & 0.004 & 15 & 16.02 & 99.52 & 27 & 5 & 14.05 & 98.64 \\
\hline 0.02 & 0.012 & 0.008 & 10 & 22.76 & 99.22 & 18 & 4 & 21.29 & 97.75 \\
\hline 0.03 & 0.018 & 0.012 & 8 & 28.13 & 98.96 & 16 & 4 & 27.27 & 96.34 \\
\hline 0.04 & 0.024 & 0.016 & 7 & 32.73 & 98.67 & 12 & 3 & 32.41 & 96.38 \\
\hline 0.05 & 0.03 & 0.02 & 6 & 36.90 & 98.56 & 12 & 3 & 36.97 & 94.85 \\
\hline 0.06 & 0.036 & 0.024 & 6 & 40.76 & 98.00 & 11 & 3 & 41.15 & 93.97 \\
\hline 0.07 & 0.042 & 0.028 & 5 & 44.17 & 98.22 & 11 & 3 & 45.03 & 92.45 \\
\hline 0.08 & 0.048 & 0.032 & 5 & 47.53 & 97.73 & 11 & 3 & 48.64 & 89.99 \\
\hline 0.002 & 0.0002 & 0.0018 & 32 & 7.52 & 99.96 & 68 & 8 & 5.63 & 99.85 \\
\hline 0.004 & 0.0004 & 0.0036 & 23 & 10.34 & 99.92 & 43 & 6 & 8.30 & 99.77 \\
\hline 0.005 & 0.0005 & 0.0045 & 21 & 11.50 & 99.90 & 39 & 6 & 9.43 & 99.72 \\
\hline 0.008 & 0.0008 & 0.0072 & 16 & 14.44 & 99.86 & 29 & 5 & 12.45 & 99.61 \\
\hline 0.009 & 0.0009 & 0.0081 & 15 & 15.30 & 99.85 & 27 & 5 & 13.36 & 99.58 \\
\hline 0.01 & 0.001 & 0.009 & 15 & 16.11 & 99.81 & 26 & 5 & 14.24 & 99.52 \\
\hline 0.02 & 0.002 & 0.018 & 10 & 22.89 & 99.71 & 17 & 4 & 21.69 & 99.23 \\
\hline 0.03 & 0.003 & 0.027 & 8 & 28.26 & 99.61 & 15 & 4 & 27.93 & 98.76 \\
\hline 0.04 & 0.004 & 0.036 & 7 & 32.85 & 99.50 & 11 & 3 & 33.23 & 98.82 \\
\hline 0.05 & 0.005 & 0.045 & 7 & 37.02 & 99.35 & 10 & 3 & 38.06 & 98.52 \\
\hline 0.06 & 0.006 & 0.054 & 6 & 40.80 & 99.25 & 10 & 3 & 42.54 & 98.03 \\
\hline 0.07 & 0.007 & 0.063 & 5 & 44.26 & 99.23 & 10 & 3 & 46.77 & 97.52 \\
\hline 0.08 & 0.008 & 0.072 & 5 & 47.62 & 99.15 & 9 & 3 & 50.74 & 97.35 \\
\hline
\end{tabular}

\section{References}

LI, C.H. 1962. A Sequential method for screening experimental variables. Jour. Amer Statist. Assoc., 57: 455-477.

MANENE, M.M. 1997. On two-type step-wise group screening designs. Proceedings of the Fifth Scientific Conference of the East, Central and Southern Africa Network of I.B.S., pp. 57-62

MANENE, M.M.; ROTICH, A.M. and SIMWA R.O. 2002. On Multi-type step-wise group screening designs. Bulletin of the Allahabad Mathematical Society, 17: 59-78. 
MAURO, C.A. and SMITH, D.E. 1982. The performance of two-stage group screening in factor screening experiments. Technom., 24: 325-330.

ODHIAMBO, J.W. 1986. The performance of multistage group screening designs. Commun. Statist. Theor. Meth., 15 (8): 2467-2481.

ODHIAMBO J.W. and MANENE M.M. 1987. Step-wise group screening designs with errors in Observations. Commun. Statist. Theor. Meth. 16(10): 3095-3115.

PATEL, M.S. 1962. Group screening with more than two stages. Technom, 4: 209-217.

PATEL, M.S. and MANENE, M.M. 1987. Step-wise group screening with equal prior probabilities and no errors in observations. Commun. Statist. Simula. and Computa, 16(3): 817-833.

STERRET, A. 1957. On the detection of defective members of large populations. Annals of Math. Statist., 28: 1033-1036.

WATSON, G.S. 1961. A study of the group screening method. Technom, 3: 371-388.

Received 28 August 2004

Accepted 5 October 2005 